\title{
Screening High-z GRBs with BAT Prompt Emission Properties
}

\author{
T. N. Ukwatta* ${ }^{*, \dagger}$, T. Sakamoto ${ }^{\dagger, * *}$, M. Stamatikos ${ }^{\dagger, *}$, N. Gehrels ${ }^{\dagger}$ and K. S. \\ Dhuga* \\ *The George Washington University, Washington, D.C. 20052 \\ ${ }^{\dagger}$ NASA Goddard Space Flight Center, Greenbelt, MD 20771 \\ ** The University of Maryland, Baltimore County, Baltimore, MD 21250 \\ ¥Oak Ridge Associated Universities, P.O. Box 117, Oak Ridge, Tennessee 37831-0117
}

\begin{abstract}
Detecting high-z GRBs is important for constraining the GRB formation rate, and tracing the history of re-ionization and metallicity of the universe. Based on the current sample of GRBs detected by Swift with known redshifts, we investigated the relationship between red-shift, and spectral and temporal characteristics, using the BAT event-by-event data. We found red-shift trends for the peak-flux-normalized temporal width T90, the light curve variance, the peak flux, and the photon index in simple power-law fit to the BAT event data. We have constructed criteria for screening GRBs with high red-shifts. This will enable us to provide a much faster alert to the GRB community of possible high-z bursts.
\end{abstract}

Keywords: Gamma-ray Bursts, High-z GRBs

PACS: 98.70.Rz, 98.62.Ai

\section{INTRODUCTION}

High-z GRBs offer the potential to probe the early universe into the epoch of reionization. In Big Bang cosmology, the universe went through a "dark age" when matter had cooled below 3000 Kelvin and became neutral. Re-ionization of matter occurred once bodies such as quasars started radiating, initiating the ionization of neutral matter, producing an ionized plasma. Bodies we see now with a red-shift $20>z>6$ (150 million to one billion years after the Big Bang) [1] emitted their radiation in this re-ionization period. (The highest-z GRB detected thus far is $\mathrm{z}=6.29$ [2].)

High-z GRBs also have the potential to trace the star formation rate and metallicity histories of the universe [1, 3]. Moreover, GRBs are 100-1000 times brighter at early times than are high red-shift quasars. GRBs are expected to occur out to $\mathrm{z}>10$, whereas the distribution of quasars drops significantly beyond $z=3$. Another benefit is that due to the relatively clean neighborhoods of GRB progenitors, GRB afterglow have simple power-law spectra with no emission lines. Thus GRBs are clean probes of the intergalactic medium (IGM), whereas quasars are contaminated by continuous material emission from the central engine to the quasar's neighborhood. High-z GRB studies will probe the IGM less than 1 Gyr after the Big Bang. In this respect, the study of high-Z GRBs provide a unique method to probe the early universe.

Clearly, detecting high-z GRBs is very important for probing the early universe. Currently, a significant number of GRBs with redshifts around $z=2$ have been observed, but only a handful of events for redshifts greater than $z=5$. To help identify new GRBs 
with high-z, we have developed an early-stage observational filter on bursts, so that follow-up ground based observatories can be notified, and they can subsequently attempt to get optical red-shift measurements for these candidate high-z objects.

\section{SCREENING HIGH-Z BURSTS USING BAT DATA}

Our observational filter for candidate high-z GRBs is a set of screening criteria, taking advantage of some prompt-emission properties of GRBs which are analyzable within a few hours after the burst.

We have found trends between some observational characteristics in the prompt emission from observed GRBs and their corresponding redshifts $(z)$ :

1. The temporal period T90, normalized by the peak photon flux (see Fig. 1);

2. The normalized light curve variance (using T100 duration) (see Fig. 2);

3. The peak photon flux (Fig. 3);

4. The photon index derived from the simple power law fit to the burst spectrum (Fig. 4).

Each characteristic is not sufficiently discriminatory to screen for high-z GRBs by their own. However, the combined cuts on these properties enable us to filter out high-z bursts with significant probability.

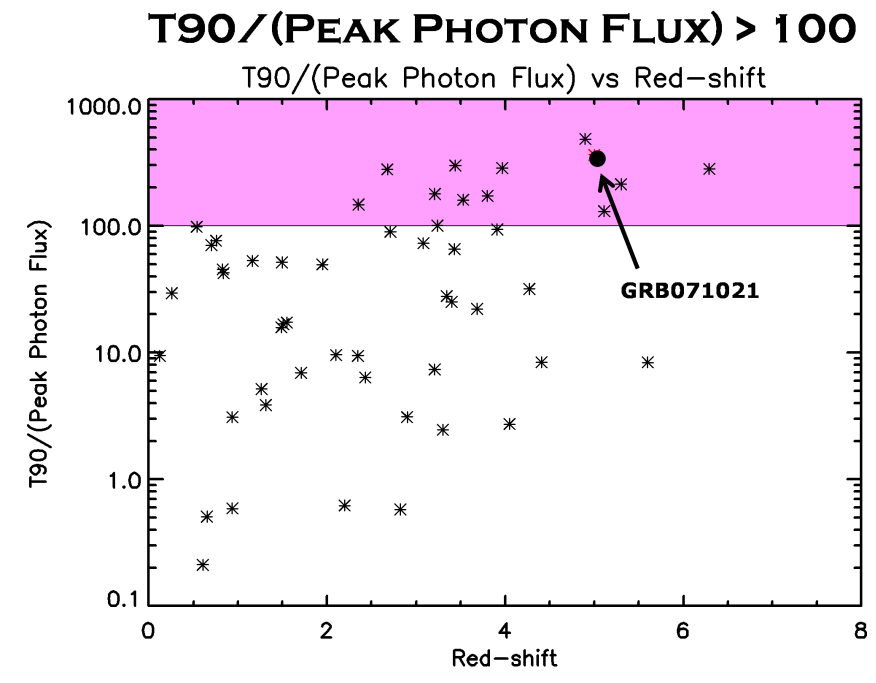

FIGURE 1. T90 normalized by the peak photon flux cut.

\section{DISCUSSION}

Using trends in pulse width, variance, peak flux, and photon power law, we have formulated automated screening criteria to identify possible high-z GRBs by utilizing the prompt emission properties of GRBs within a few hours after the burst. This will enable more intense ground-based observational campaigns for measuring red-shifts of the detected GRBs. 
With the limited available sample of GRBs having known high-z, the probability of a GRB being selected by our criteria with a redshift $\geq 5.0$ is $45 \%$, with a redshift $\geq 4.0$ is $67 \%$ and with a redshift $\geq 3.5$ is $89 \%$ (Table 1). Based on an analysis of Swift existing burst data, the criteria we used will generate an alert once a month on average.

Currently, our screening criteria are implemented in automated scripts and running in real time. Our filter for high-z has already generated alerts on GRB 071021 (GCN 6967), GRB 071118 (GCN 7109) and GRB 071129 (GCN 7139), which enhanced ground based follow-up observations.

We are working on implementing these screening criteria to BAT TDRSS data. We also note that there is an ongoing effort to formulate high-z screening criteria to include the XRT and UVOT datasets.

\section{LIGHT CURVE VARIANCE $<0.0001$}

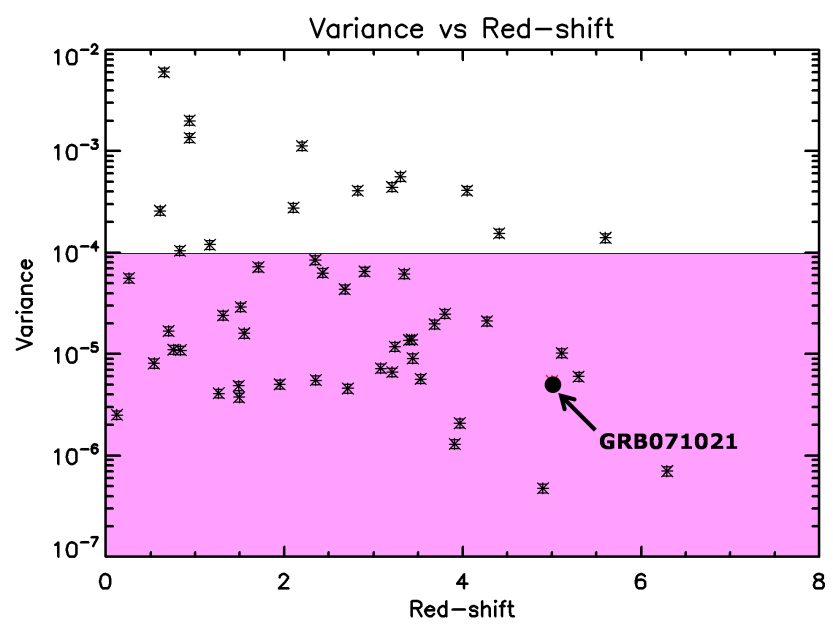

FIGURE 2. Normalized light curve variance cut.

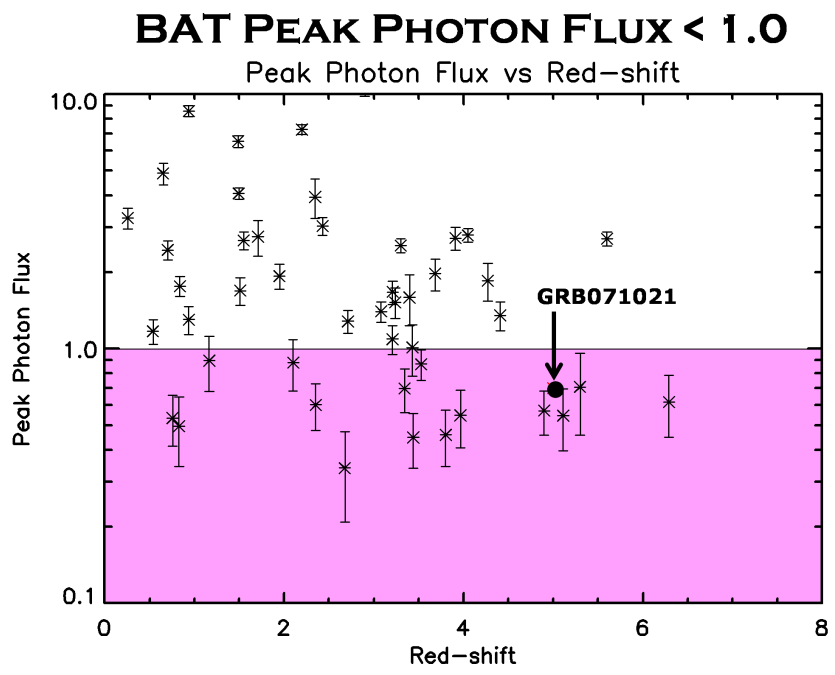

FIGURE 3. BAT peak photon flux cut. 
TABLE 1. Selected GRBs using the high-z criteria from Swift GRBs with known red-shifts.

\begin{tabular}{lr} 
Gamma-ray Burst Name & Measured Red-shift \\
\hline GRB 050730 & 3.967 \\
GRB 050814 & 5.3 \\
GRB 050904 & 6.29 \\
GRB 060115 & 3.53 \\
GRB 060510B & 4.90 \\
GRB 060522 & 5.11 \\
GRB 060605 & 3.8 \\
GRB 061110B & 3.44 \\
GRB 070110 & 2.352 \\
\hline
\end{tabular}

\section{POWER LAW PHOTON INDEX > -2.0}

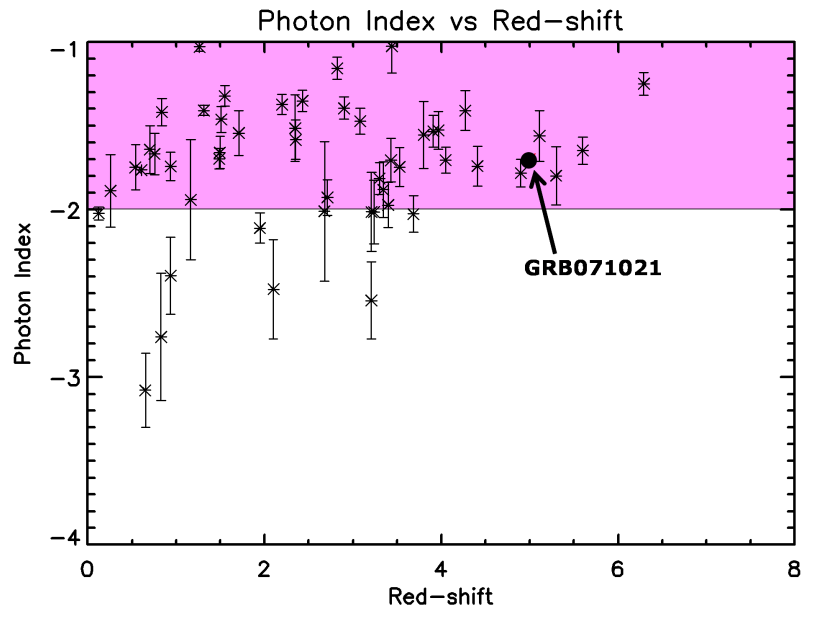

FIGURE 4. Photon Index cut.

\section{REFERENCES}

1. D. Q. Lamb, and D. E. Reichart, ApJ, 2000, 536, 1

2. G. Tagliaferri et al., A\&A, 2005, 443, L1

3. M. Gilfanov, R. Sunyeav, and E. Churazov, Proc. of the MPA/ESO/MPE/USM Joint Astronomy Conference, 2002, 157 